BULL. AUSTRAL. MATH. SOC.

VOL. $29(1984), 151-165$.

\title{
COLORING ISLAND MAPS
}

\section{Brad Jackson and Gerhard Ringel}

\begin{abstract}
An empire map is a map where the set of countries is divided into disjoint subsets which we call empires. If one empire consists of a single country then the remaining map is called an empire island map, the single country being referred to as the ocean. If every empire consists of at most $M$ countries then it is called an $M$-pire map.

It is proved that an $M$-pire map is colorable by $6 M-2$ colors. Further there exists such a map which has $6 M-2$ mutually adjacent empires.

If an $M$-pire island map has every country adjacent to the ocean, then it is colorable in $4 M$ colors. Such a map exists with $4 M$ mutually adjacent empires where every country is adjacent to the ocean.
\end{abstract}

In this paper we deal with coloring maps. For uniformity we always call a single face of a map, a country. Two countries are said to be adjacent if they share a common boundary edge. To properly color a map one should assign different colors to adjacent countries. A map is said to be $k$-colorable if it can be properly colored using $k$ colors. For any set of maps $S$ (possibly one map), the chromatic number of $S$ is defined to be the smallest number $k$, such that every map in $S$ is $k$-colorable. The

Received 5 October 1983.

Copyright Clearance Centre, Inc. Serial-fee code: 0004-9727/84 $\$ A 2.00+0.00$. 
famous four color theorem states that four is the chromatic number of the set of planar maps. In this paper we consider colorings of a special kind of maps, which we call island maps.

In [1] Gardner popularized a generalization by Heawood of the four color theorem to maps of empires. An empire map is a map where the set of countries is divided into disjoint subsets which we call empires. If we wish to specify an empire with exactly $m$ components we call it an m-pire. To properly color an empire map one must assign one color to each empire so that again adjacent countries of distinct empires are assigned different colors. An empire map where each empire has $m \leq M$ components is called an $M$-pire map. Define $\chi(M)$ to be the chromatic number of the set of planar $M$-pire maps. Thus $\chi(1)=4$ by the four color theorem. Heawood [2] was the first to show that $\chi(M) \geq 6 M$ for all $M \leq 2$. He failed to verify that equality holds except for $M=2$ which he did by exhibiting a planar map of 12 mutually adjacent 2-pires. In general it has been shown [1], [2] by Heawood and Taylor that $\chi(M)=6 M$ for $M=2,3,4$, but $\chi(M)$ is unknown for $M \geq 5$.

Now we consider a special kind of empire map on the plane (sphere) that has one empire consisting of a single country $x$ to which every other empire is adjacent. Disregarding $x$ we call the remaining map, an empire island map, and sometimes we call $x$ the ocean. See Figures 1-6 for examples of empire island maps. We color these maps just like regular empire maps except that the ocean is not colored. We will prove the following four theorems about coloring empire island maps.

THEOREM 1. Any M-pire is zand map is colorable by $6 M-2$ colors.

THEOREM 2. Let I be an M-pire island map where every country is adjacent to the ocean. Then $I$ is colorable by $4 M$ colors.

THEOREM 3. For every $M \geq 2$, there exists an $M$-pire island map of $6 M-2$ mutually adjacent empires.

THEOREM 4. For every $M \geq 2$, there exists an $M$-pire island map of $4 M$ mutually adjacent empires with every country adjacent to the ocean.

Figures 1-3 give examples of $M$-pire island maps of $6 M-2$ mutually adjacent empires for $M=2, M=3$, and $M=4$. Theorems 1 and 3 together will be enough to show that the chromatic number of the set of 


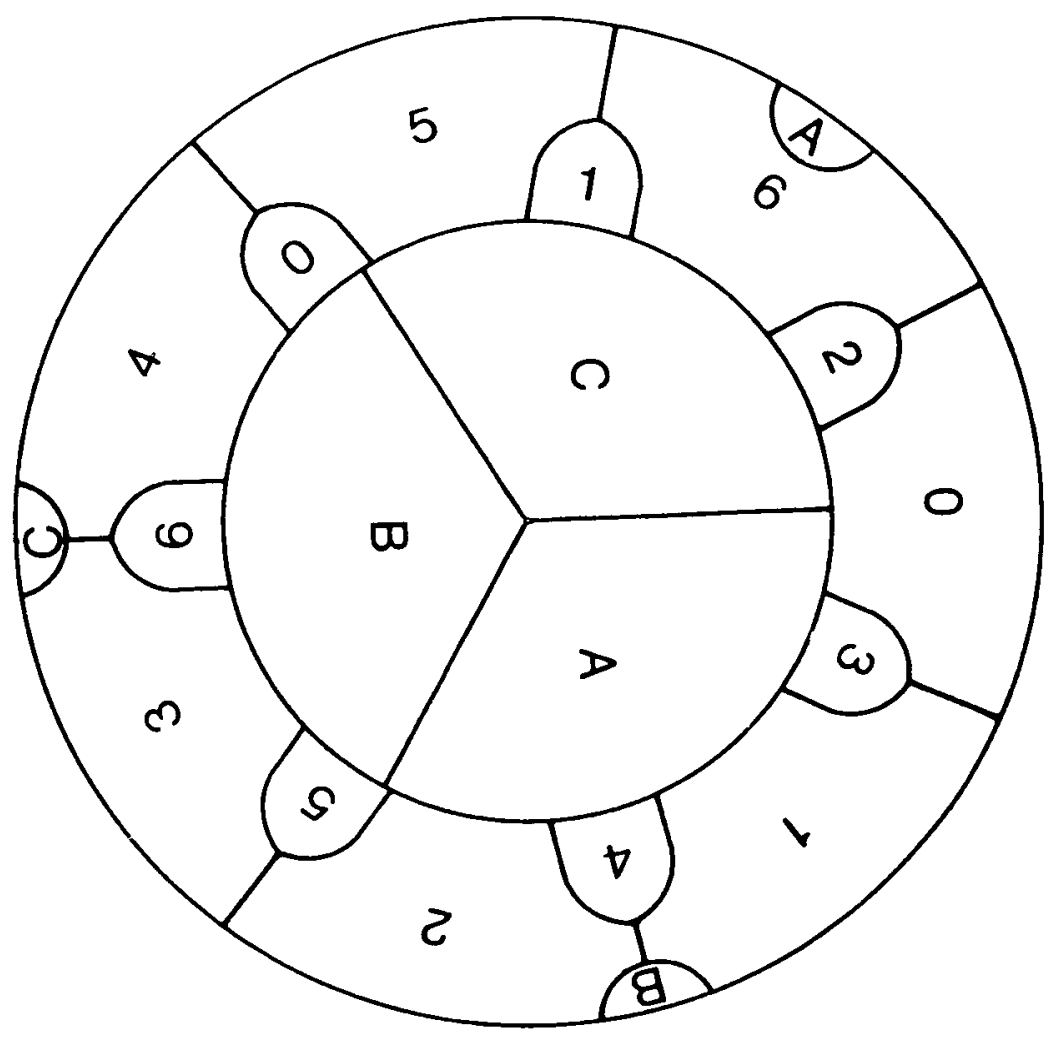

FIGURE 1 


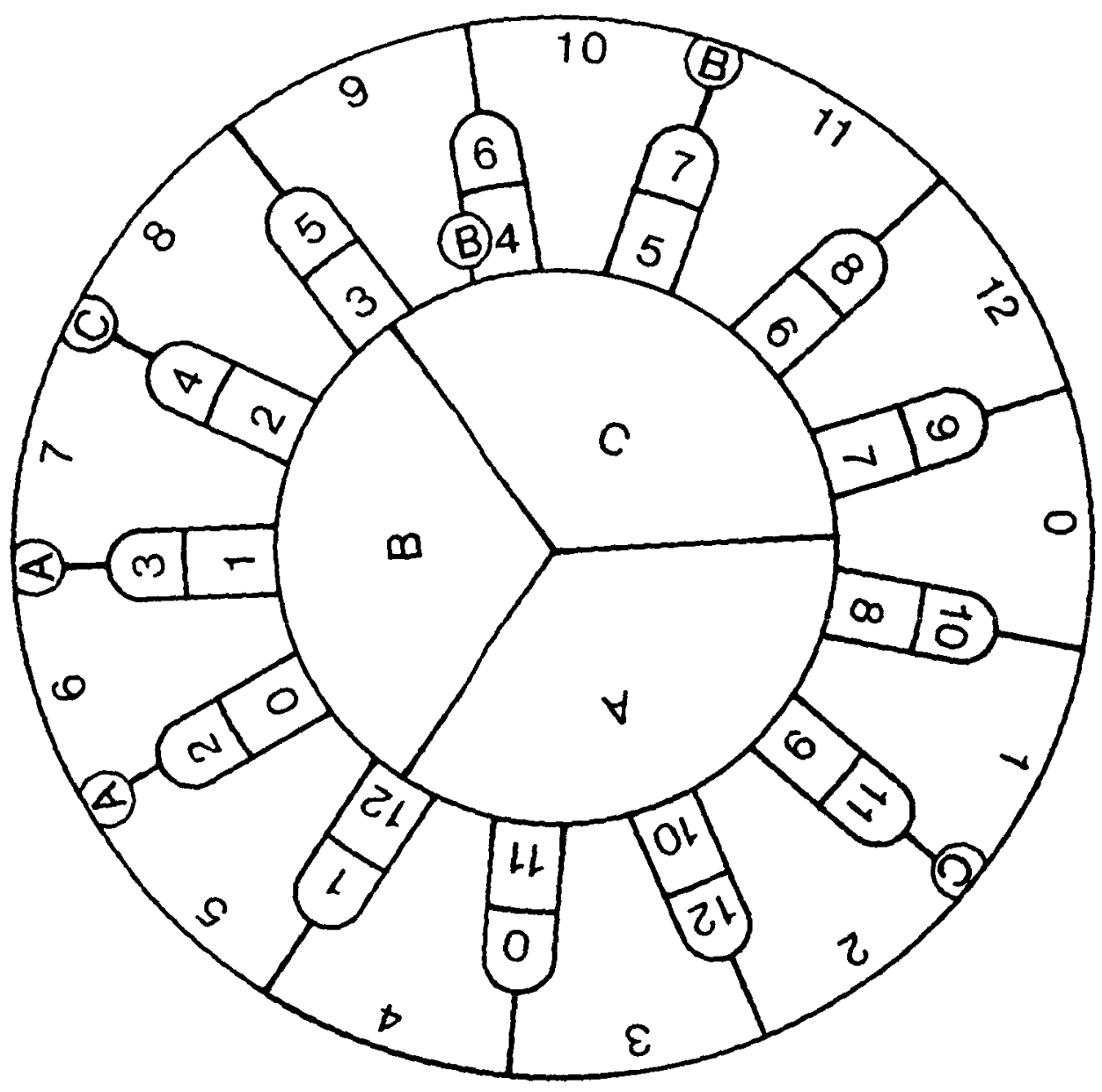

FIGURE 2 


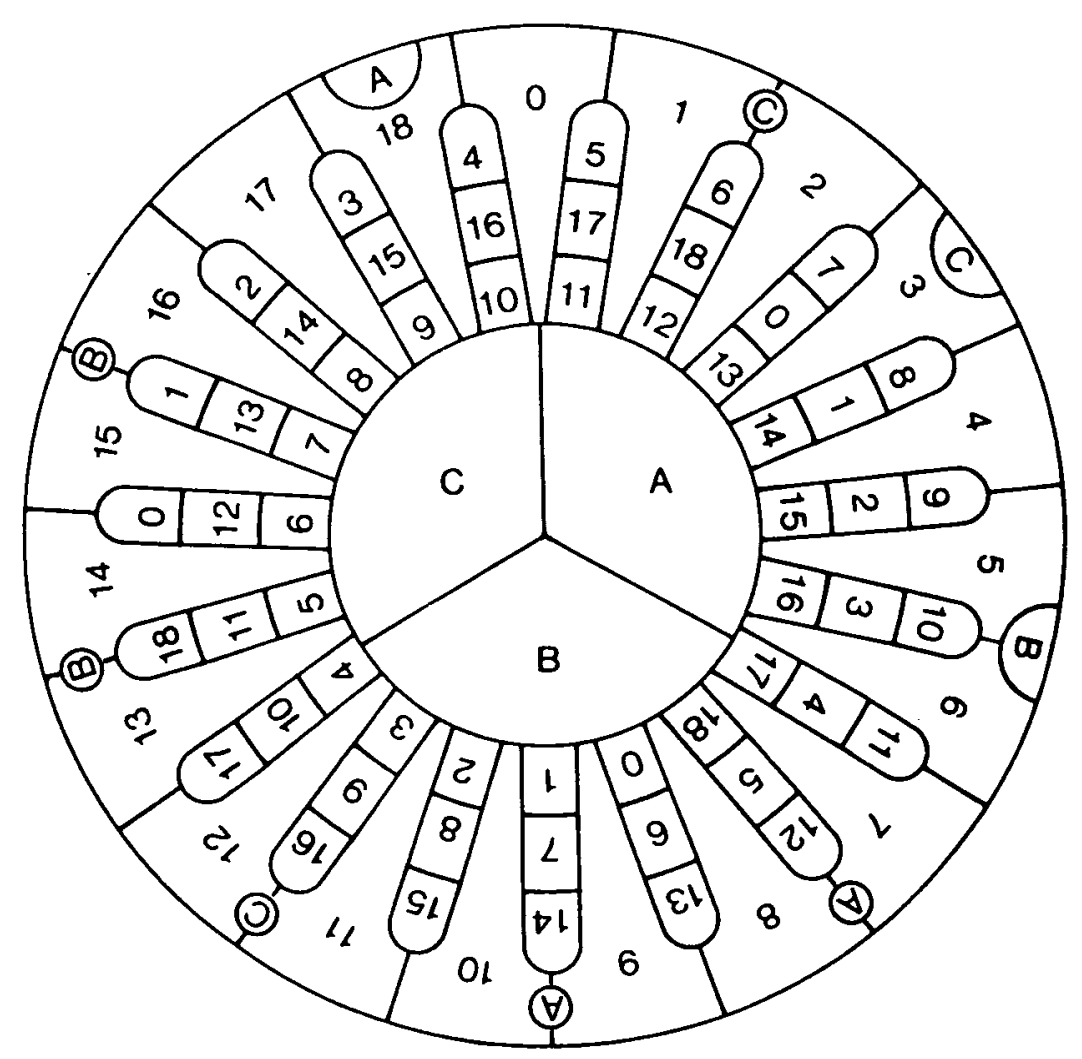

FIGURE 3 


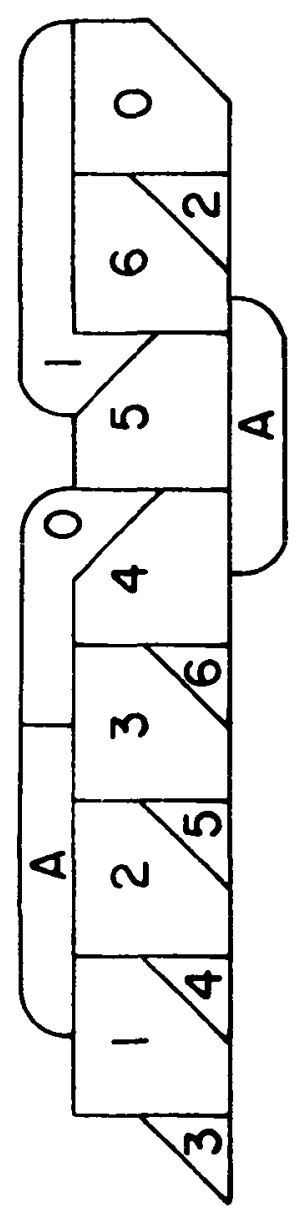

올 


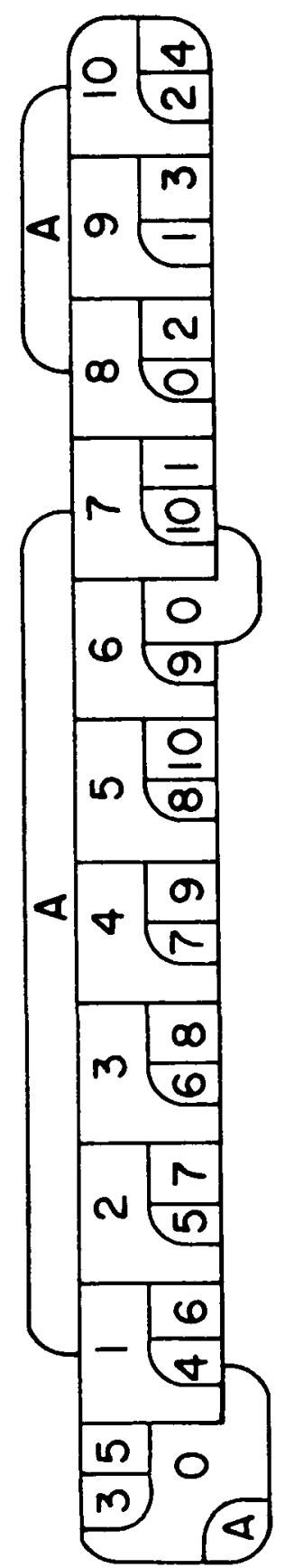

을 


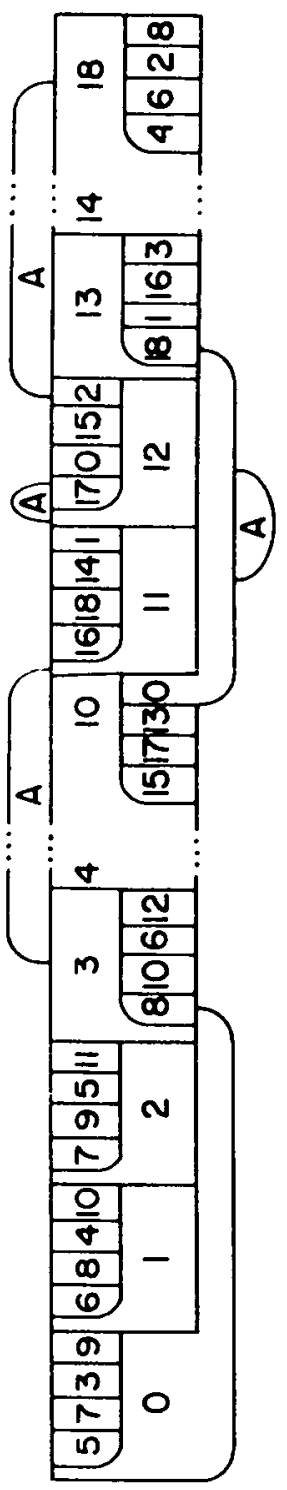

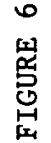


$M$-pire island maps for $M \geq 2$, is exactly $6 M-2$. If $M=1$ it is easy to show that the chromatic number is 3 , even without using the proof of the four color theorem.

Figures 4-6 give examples of $M$-pire island maps of $4 M$ mutually adjacent empires where every country is adjacent to the ocean for $M=2,3$, and 4 . Theorems 2 and 4 together will show that the chromatic number of the set of $M$-pire island maps with every country touching the ocean is exactly $4 M$.

Proofs of Theorems 1 and 2. Without loss of generality we need only consider island maps where any country is adjacent to the ocean along at most one edge. We define the degree of a country $C$ in a map to be the number of countries to which $C$ is adjacent. The degree of an empire is the sum of the degrees of all the countries in that empire. As a consequence of Euler's formula for a map on the plane (sphere) with $c$ countries one can say that the sum of the degrees of all the countries is at most $6 c-12$.

Let $I$ be an $M$-pire island map of $n$ empires and $C \leq n M$ countries. Let $I^{*}$ be the same map counting the ocean as an empire with a single country. Then in $I^{*}$ let $d_{1}, d_{2}, \ldots, d_{c}$ be the degrees of the original countries and let $d_{0}$ be the degree of the ocean. Since $I^{*}$ is a map on the plane then

$$
d_{1}+d_{2}+\ldots+d_{c}+d_{0} \leq 6(c+1)-12=6 c-6 .
$$

First suppose that in $I^{*}$ every country is adjacent to the ocean, thus $d_{0} \leq c$. In $I$ the degree of every country is reduced by one. So if $e_{1}, e_{2}, \ldots, e_{n}$ are the degrees of the empires in $I$ then we have

$$
e_{1}+e_{2}+\ldots+e_{n} \leq 4 c-6 \leq 4 n M-6
$$

so there exists some empire in $I$ whose degree is less than $4 M$.

For an arbitrary island map the only requirement is that each empire is adjacent to the ocean in $I^{*}$, thus $d_{0} \geq n$ and in $I$ the degree of every other empire is reduced by at least one. So in this case we have

$$
e_{1}+e_{2}+\ldots+e_{n} \leq 6 c-6-2 n \leq(6 i n-2) n-6
$$


so there exists some empire in $I$ whose degree is less than $6 M-2$.

A new map can be formed from any map by shrinking any country to a point. In this same way an entire empire can be removed from the map. To complete the proofs of Theorems $I$ and 2 we only need the following lemma.

LEMMA. Let $S$ be a set of empire maps which is closed under the removal of empires. If every map in $S$ has some empire whose degree is less than $D$, then every map in $S$ is colorable by $D$ colors.

Suppose this is not the case. Then there is an empire map $I$, which is not $D$-colorable, and with the least number of empires of any such map in $S$. From the map $I$ we could remove an empire $E$ with degree less than $D$ and color the resulting map with $D$ colors. In $I$ at least one of the $D$ colors is not used on an empire adjacent to $E$. We obtain a proper $D$-coloring of $I$ by coloring $E$ with one of these missing colors. But this is a contradiction since $I$ was not $D$-colorable. This completes the proofs of Theorems 1 and 2 .

Proof of Theorem 3. If you glance at the maps in Figures 1-3 you will notice that the numbered empires form a symmetrical ring and that there are three more empires $A, B, C$ added. First we show how to construct the symmetrical ring of $6 M-5$ mutually adjacent $M$-pires.

Consider the current graph in Figure 7 with currents in the group $\mathbb{Z}_{19}$ and in general the current graphs in Figure 8 or 9 with currents in the group $\mathbb{Z}_{12 s+1}$ or $\mathbb{Z}_{12 s+7}$ respectively, depending on whether $M=2 s+1$ is odd or $M=2 s+2$ is even. In these current graphs the following properties are crucial. Each edge of the graph has a current which is an element of the appropriate group and

(Cl) the currents together with their inverses contain every nonzero element of the group exactly once;

(c2) at each vertex of valence 3 the sum of the inward flowing currents equals the sum of the outward flowing currents.

For simplicity we prefer to have the element 1 as the current on the right most edge of the current graph. So we multiply each current in Figures $7-9$ by 6 to obtain new current graphs with 1 as the current on the right most edge. For example the current graph obtained from Figure 7 


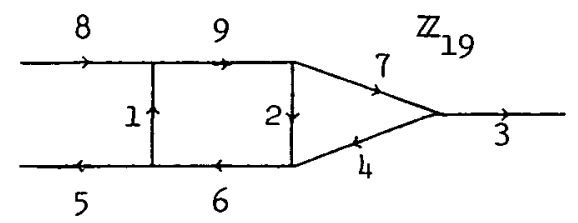

FIGURE 7

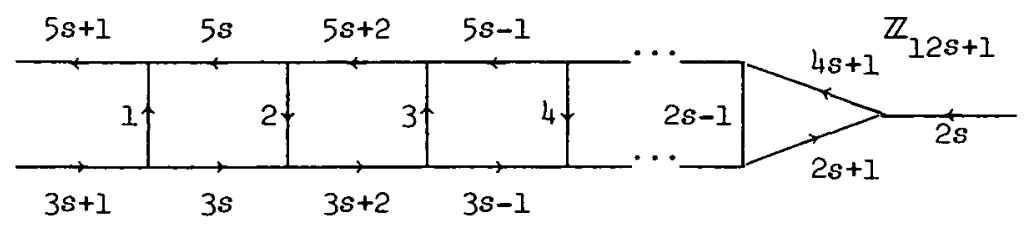

FIGURE 8

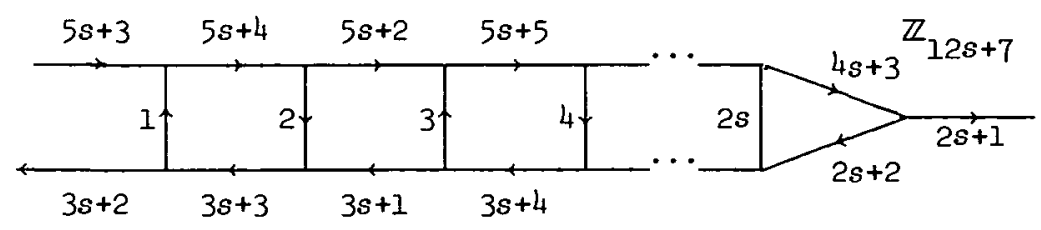

FIGURE 9

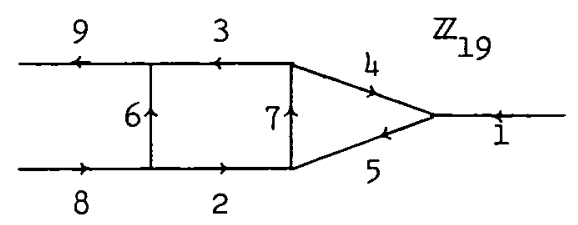

FIGURE 10

is shown in Figure 10. Since 6 is relatively prime to the modulus of the group one can easily show that properties (C1) and (C2) still hold in these new current graphs.

Also notice that in Figure 8 the two currents on the leftmost edges become $6 s-4$ and $6 s-3$ and the two currents on the leftmost edges in Figure 9 become $6 s+2$ and $6 s+3$.

To start with we embed these current graphs onto the disk dividing it into $M+1$ regions, one triangle, $M-3$ quadrilaterals, and 3 "exterior" regions. For $M=4$ this is pictured in Figure 11 . 


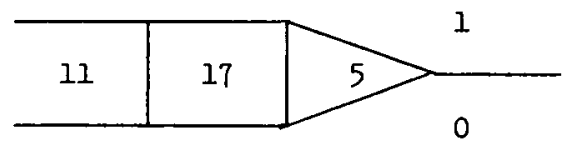

FIGURE 11

Next we label the regions with elements of the group. We start by labelling the bottom exterior region 0 . We continue this labelling in the following manner. If a region labelled $x$ is adjacent to another region along a boundary edge with current $c$, in the counter clockwise (respectively clockwise) direction along the boundary of $x$, then the adjacent region is labelled $x+c$ (respectively $x-c$ ). Since property (C2) holds this leads to the unique labelling, in Figure 1l. For any nonzero element $z \in \mathbb{Z}_{19}$ we obtain a new figure by adding $z$ to each of the labels in Figure 11. These 19 figures can be joined along the top and bottom exterior regions to obtain the symmetric ring of 19 4-pires in Figure 3. They are mutually adjacent because of property ( $\mathrm{Cl}$ ). In general we can obtain a ring of $6 M-5$ mutually adjacent $M$-pires in the same manner from the current graphs in Figures 8 and 9.

To obtain an $M$-pire island map of $6 M-2$ mutually adjacent empires we now add three extra empires $A, B, C$. Along the inner ring (see Figure 3) we have $12 M-10$ countries. We can divide the inner region into three "almost equal" parts $A, B$, and $C$ which are adjacent to $4 M-2,4 M-3$, and $4 M-2$ countries respectively. Since the leftmost currents of the current graphs are sufficiently large we get no repeated numbers in each of these three groups.

So far $A$ and $C$ are not adjacent to $2 M-3$ numbered empires and to the ocean which is the region outside the ring and $B$ is not adjacent to $2 M-2$ numbered empires and the ocean. There are at least two successively numbered empires to which $B$ is not adjacent. There is a vertex in the map where these two numbered empires and the ocean meet. Around this point we can add a triangular country labelled $B$ which is adjacent to all three. The remaining $2 M-4$ adjacencies can be created by adding $M-2$ lunes labelled $B$ in the middles of appropriate edges. Similarly one can add $M-1$ lunes to obtain the needed adjacencies for empires $A$ and $C$. Thus we get $6 M-2$ mutually adjacent $M$-pires so 
that each empire is adjacent to the ocean, completing the proof of Theorem 3.

Proof of Theorem 4. A similar construction will give us maps of $4 M$ mutually adjacent empires where each country is adjacent to the ocean. We start with the current graphs in Figures 12 and 13 with currents in the groups $\mathbb{Z}_{8 s+3}$ and $\mathbb{Z}_{8 s+7}$ depending on whether $M=2 s+1$ and is odd or $M=2 s+2$ and is even. The current graphs also satisfy the crucial properties (Cl) and (C2). Again we embed the current graph in the disk and get $M+1$ labelled regions as before starting with 0 as the label of the upper leftmost region and $l$ as the label of the rightmost region. For every nonzero $\boldsymbol{z} \in \mathbb{Z}_{4 M-1}$ we obtain a new disk by adding $\boldsymbol{z}$ to all the labels in the original disk. Thus we get a total of $4 M-1$ such regions.

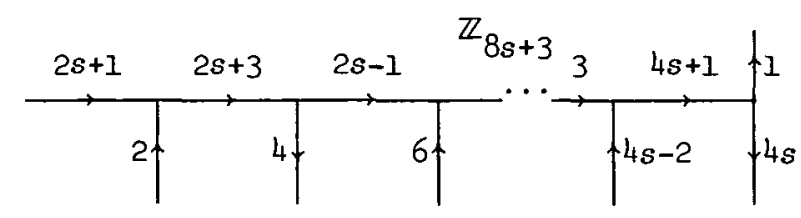

FIGURE 12

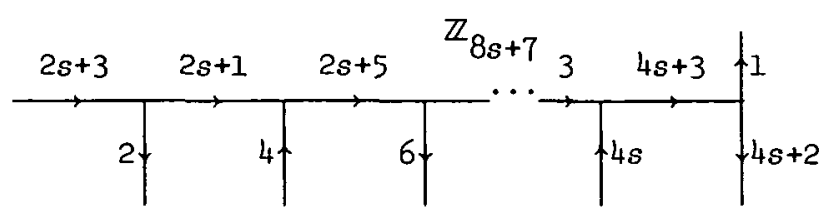

FIGURE 13

We merge these regions into a line by identifying the rightmost and leftmost regions labelled $1,2,3, \ldots, 4 M-2$. Every empire has $M$ components except for empire 0 , which has $M+1$ components, so we delete the rightmost country of empire 0 which is adjacent to countries $4 M-2$ and $2 M-2$. Notice that we can identify each region in the upright position (with the edge of current one on top) or in the flipped position (with the edge of current one on the bottom). We identify the disks with leftmost regions $2 s-1,2 s, \ldots, 2 M, 2 M+2 s-1,2 M+2, \ldots, 4 M-2$ in the upright position and all others in the flipped position.

To recreate our two missing adjacencies we extend the rightmost 
country of empire 0 along the bottom of the line of numbered empires creating an adjacency between empire 0 and empire $2 M-2$ in addition to extra adjacencies between empire 0 and empires $2,3, \ldots, 2 s-1$. Finally we create the last needed adjacency by extending country 0 , in the disk with leftmost region labelled $2 M$, along the bottom until it touches country $4 M-2$, in the disk with leftmost region labelled $2 M+2 s-1$, also creating extra adjacencies between empire 0 and empires $2 M+2, \ldots, 2 M+2 s-1$. Figures 5 and 6 picture this situation for $M=3$, and $M=5$. Thus we obtain $4 M-1$ mutually adjacent $M$-pires. The construction for $M=2$ does not fit the general pattern but is given in Figure 4. To finish the construction we add an extra empire $A$ which is adjacent to each of the numbered empires. Along the top of the line of numbered empires we add two components of $A$, one adjacent to empires $2 s-1,2 s, \ldots, 2 M+1$ and one adjacent to empires $2 s-2,2 M+2 s-1,2 M+2 s, \ldots, 4 M-2(2 M+2 s-1, \ldots, 4 M-2$, if $s=1)$. We then add triangular countries labelled $A$ along the ocean adjacent to empires 1 and $2 M+2,2$ and $2 M+3, \ldots, 2 s-3$ and $2 M+2 s-2$, and one lune adjacent to empire 0 and the ocean. Since $A$ has $2 s$ components ( 3 , if $s=1$ ) we have constructed an $M$-pire island map of $4 M$ mutually adjacent empires so that each country is adjacent to the ocean, completing the proof of Theorem 4.

We conclude by mentioning one unsolved problem involving island maps. We define an $M$-archipelago map to be a collection of $M$ island maps in one ocean such that every empire has at most one component on any island. Even for $M=2$ the chromatic number for $M$-archipelago maps is unknown. One can construct a 2-archipelago map of 6 mutually adjacent 2-pires but it is impossible to do so with 7 mutually adjacent 2-pires. As before we can show that any 2-archipelago map is 8-colorable. So all we know is that 6,7 , or 8 is the chromatic number for 2-archipelago maps.

\section{References}

[1] Martin Gardner, "Mathematical games", Sci. Amer. (1980), 14. 
[2] P.J. Heawood, "Map-colour theorem", Quart. J. Pure App Z. Math. 24 $(1890), 332-338$.

Department of Mathematics,

Division of Natural Sciences,

University of California,

Santa Cruz,

California 95064 ,

USA. 